\title{
11/-hydroxysteroid dehydrogenase-1 deficiency alters the gut microbiome response to Western diet
}

\author{
Jethro S Johnson', Monica N Opiyo², Marian Thomson ${ }^{3}$, Karim Gharbi³, \\ Jonathan R Seckl2 ${ }^{2}$, Andreas Heger1,* and Karen E Chapman ${ }^{2, *}$ \\ ${ }^{1}$ Computational Genomics Analysis and Training, Medical Research Council-Functional Genomics Unit, \\ Department of Physiology, Anatomy and Genetics, University of Oxford, Oxford, UK \\ 2University/BHF Centre for Cardiovascular Science, Queen's Medical Research Institute, Edinburgh, UK \\ ${ }^{3}$ Edinburgh Genomics, Ashworth Laboratories, University of Edinburgh, Edinburgh, UK \\ *(A Heger and K E Chapman contributed equally to this work)
}

Correspondence should be addressed

to K E Chapman

Email

karen.chapman@ed.ac.uk

\begin{abstract}

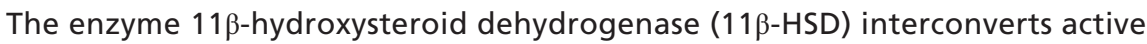

glucocorticoids and their intrinsically inert 11-keto forms. The type 1 isozyme, 11 $\beta$-HSD1, predominantly reactivates glucocorticoids in vivo and can also metabolise bile acids.

$11 \beta$-HSD1-deficient mice show altered inflammatory responses and are protected against the adverse metabolic effects of a high-fat diet. However, the impact of $11 \beta-H S D 1$ on the composition of the gut microbiome has not previously been investigated. We used high-throughput $16 \mathrm{~S}$ rDNA amplicon sequencing to characterise the gut microbiome of $11 \beta$-HSD1-deficient and C57BI/6 control mice, fed either a standard chow diet or a cholesterol- and fat-enriched 'Western' diet. 11 $\beta$-HSD1 deficiency significantly altered the composition of the gut microbiome, and did so in a diet-specific manner. On a Western diet, $11 \beta$-HSD 1 deficiency increased the relative abundance of the family Bacteroidaceae, and on a chow diet, it altered relative abundance of the family Prevotellaceae. Our results demonstrate that (i) genetic effects on host-microbiome interactions can depend upon diet and (ii) that alterations in the composition of the gut microbiome may contribute to the aspects of the metabolic and/or inflammatory phenotype observed with $11 \beta$-HSD 1 deficiency.
\end{abstract}

\author{
Key Words \\ - glucocorticoids

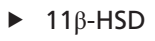 \\ $\checkmark$ diet \\ - gut microbiome
}

Journal of Endocrinology (2017) 232, 273-283

\section{Introduction}

The gut is increasingly recognised as an important source of bacterially derived signals that have the potential to influence host physiology, including via the endocrine system (Evans et al. 2013, Brown \& Hazen 2015). Conversely, dietary or other metabolic perturbations can alter the composition of the gut microbiota in a manner that correlates with disease states (Giongo et al. 2011). Although increased understanding of these host-microbiome interactions promises to identify many novel therapeutic targets for the prevention and treatment of disease, at present, the molecular mechanisms that underpin such interactions remain largely uncharacterised.

The enzyme 11ß-hydroxysteroid dehydrogenase type 1 (11ß-HSD1) contributes to intracellular glucocorticoid action by catalysing the conversion of inactive to active forms of the principal glucocorticoids found in humans (cortisone to cortisol) and mice 
(11-dehydrocorticosterone to corticosterone). Whilst widely distributed, 11 $\beta$-HSD1 regulates intracellular glucocorticoid levels in a localised and tissue-specific manner, largely independent of circulating cortisol/ corticosterone levels (Chapman et al. 2013). It is therefore an important contributor to glucocorticoid modulation of immunological and metabolic pathways. Tissue levels of $11 \beta$-HSD1 are elevated in adipose tissue in metabolic disease and in hippocampus in age-related cognitive decline. Inhibition of $11 \beta$-HSD 1 is a potential therapeutic target for treatment of a range of conditions, including type 2 diabetes-metabolic syndrome, atheromatous coronary artery disease and age-related cognitive decline (Anderson \& Walker 2013, Chapman et al. 2013).

In mice, inhibition of, or deficiency in, $11 \beta$-HSD1 is protective against the adverse metabolic and pro-inflammatory effects of a high-fat diet (Morton et al. 2004, Wamil et al. 2011) and improves insulin sensitivity/glucose homeostasis in models of type 2 diabetes (Morton et al. 2004). 11ß-HSD1 deficiency/inhibition also reduces atherosclerosis in atherosclerosis-prone Apoe $e^{-/-}$mice fed a cholesterol and fat-enriched 'Western' diet, attributed in part to reduced cholesterol accumulation in macrophages (García et al. 2013, Kipari et al. 2013). Consistent with the widely recognised role of glucocorticoids within the immune system, 11 $\beta$-HSD1 deficiency or inhibition in mice increases the severity of acute inflammatory responses (Coutinho et al. 2012) and modulates macrophage phenotype (Gilmour et al. 2006, Zhang \& Daynes 2007, McSweeney et al. 2010), the latter, potentially an important determinant of gut microbial composition (Wu \& Wu 2012). However, the relationship between $11 \beta$-HSD1 activity and composition of the gut microbiota has not been examined.

In addition, there is increasing evidence that $11 \beta$-HSD1 both influences and is, in turn, directly influenced by bile acid homeostasis. Bile acids, produced, like steroid hormones, from cholesterol, are inhibitors of $11 \beta$-HSD1 and some secondary bile acids (generated via gut bacterial activity) are directly metabolised by $11 \beta$-HSD 1 (Perschel et al. 1991, Buhler et al. 1994, Odermatt et al. 2011, Penno et al. 2013, 2014). Importantly, the ability of bile acids to alter the gut microbiome has been demonstrated (Islam et al. 2011, Yokota et al. 2012), with potential clinical implications (Buffie et al. 2015). Thus, $11 \beta$-HSD1 has the potential to affect the microbiome via bile acid-mediated host-microbiome cross-talk as well as through glucocorticoid-regulated immune pathways.
In this study, we test the hypothesis that $11 \beta-H S D 1$ influences the composition of the gut microbiome in a diet-dependent manner. To this end, we used high-throughput sequencing of amplified 16S rRNA gene sequences to quantify the composition of the gut microbiota from $H s d 11 b 1^{\text {Del1/Dell }}$ mice, with global knock-out of 11ß-HSD1, and control C57Bl/6 mice. To explore possible interactions among 11 $\beta$-HSD 1 activity, the gut microbiome and diet, comparison between genotypes was made using mice fed on standard chow diet or on a high-fat/cholesterol-enriched 'Western' diet.

\section{Materials and methods}

\section{Animals}

Animal studies were carried out in strict accordance with the UK Home Office Animals (Scientific Procedures) Act, 1986 following approval by the University of Edinburgh Animal Welfare and Ethical Review Body. Hsd $11 b 1^{\mathrm{f} / \mathrm{f}}$ mice (with a 'floxed' allele of the Hsd11b1 gene) were generated by Artemis Pharmaceuticals (Cologne, Germany) onto a C57Bl/6 background (White et al. 2015). LoxP sites were placed flanking exon 3 of the mouse $H s d 11 b 1$ gene, excision of which results in a null allele (here termed Del1) by out of frame splicing from exon 2 to exon 4 . Hsd11b1Del1/Dell mice were generated by crossing $H s d 11 b 1^{1 / f}$ mice with Hprt-Cre transgenic mice (Tang et al. 2002), resulting in germline disruption of the $H s d 11 b 1$ gene. $\mathrm{Cre}^{+}$offspring were bred with $\mathrm{C} 57 \mathrm{Bl} / 6$ and $\mathrm{Cre}^{-}$offspring in which germline deletion of exon 3 of the $H s d 11 b 1$ gene had occurred were backcrossed to $\mathrm{C} 57 \mathrm{Bl} / 6$ for $>5$ generations. Animals were fed with standard chow (unless stated otherwise) and water ad libitum. Lights were on from 07:00 to 19:00, and temperature was maintained at $22^{\circ} \mathrm{C}$. To avoid inter-animal variability that might be introduced due to differences in the stage of estrous in females, male mice were used in experiments.

Male Hsd11b1Del1/Dell mice and age-matched C57Bl/6 controls (10 weeks old) were singly housed in metabolic cages. Mice were either fed on standard chow (CRM diet: SDS, UK) or Western diet (WD, 41\% fat with $\sim 0.2 \%$ cholesterol; D12079B; Research Diets Inc., New Brunswick, NJ, USA) ad libitum, for 2 weeks $(n=6 /$ group). Neither diet was associated with significant weight gain, measured over the second week (2-way ANOVA of body weight by genotype/time, chow diet: $P=0.06$, Western diet: $P=0.8$. Body weight gain on chow diet, C57Bl/6: $1.52 \pm 0.23 \mathrm{~g}$ and Hsd11b1 Del/Del1, $1.19 \pm 0.42 \mathrm{~g}$. http://joe.endocrinology-journals.org DOI: $10.1530 / J O E-16-0578$
(C) 2017 The authors Printed in Great Britain
Published by Bioscientifica Ltd 
Body weight gain on Western diet: C57Bl/6: $-0.33 \pm 0.21 \mathrm{~g}$ and $\left.H s d 11 b 1^{\text {Del1/Del } 1},-0.18 \pm 0.25 \mathrm{~g}\right)$. All mice were killed by $\mathrm{CO}_{2}$ asphyxiation after a 4-h fast (08:00-12:00), and the contents of the caecum and colon were collected and frozen for subsequent DNA extraction.

\section{DNA extraction and sequencing}

Genomic DNA was extracted from colon and caecal contents using a QIAamp DNA stool mini kit (with proteinase $\mathrm{K}$ used for lysis), as directed by the manufacturer (Qiagen). Extracted DNA was adjusted to $10 \mathrm{ng} / \mu \mathrm{L}$ and PCR amplified in duplicate (to control for PCR bias and error), following the protocol of Caporaso and coworkers (Caporaso et al. 2012) to target the variable V4 region of the $16 \mathrm{~S}$ rRNA gene. PCR reactions $(25 \mu \mathrm{L})$ contained $10 \mu \mathrm{L}$ input DNA, $12.5 \mu \mathrm{L}$ KAPA HiFi HS Ready Mix (Kapa Biosystems, Ltd, London, UK), 16S amplicon forward primer (5 pmol, Integrated DNA Technologies, Leuven, Belgium), 16S amplicon Golay barcoded reverse primer (5 pmol, Integrated DNA Technologies) and $1.0 \mu \mathrm{L} \mathrm{H}_{2} \mathrm{O}$. PCR comprised an initial incubation at $98^{\circ} \mathrm{C}$ for $3 \mathrm{~min}$ followed by 20 cycles of $95^{\circ} \mathrm{C}$ for $20 \mathrm{~s}$, $60^{\circ} \mathrm{C}$ for $30 \mathrm{~s}, 72^{\circ} \mathrm{C}$ for $45 \mathrm{~s}$ and a final extension of $72^{\circ} \mathrm{C}$ for $10 \mathrm{~min}$. After amplification, the PCR products were diluted to $50 \mu \mathrm{L}$ with $10 \mathrm{mM}$ Tris- $\mathrm{HCl} \mathrm{pH} 8.5$ and cleaned using Ampure XP beads (Beckman Coulter Life Sciences, High Wycombe, UK) using a 1:1 bead to DNA ratio to remove excess primers, primer dimers and PCR reagents. Quality of amplicon libraries was assessed on a Bioanalyser High Sensitivity chip (Agilent Technologies) and quantification was done by qPCR using Kapa Library Quantification Kit for Illumina platforms (Kapa Biosystems, Ltd). Libraries were normalised and pooled in equimolar proportions prior to sequencing duplicate samples on an Illumina MiSeq platform using 150 base paired-end reads (v2 chemistry).

\section{Sequence assembly, filtering and taxonomic assignment}

Paired-end reads were assembled into single contiguous sequences and filtered using Mothur (Schloss et al. 2009) (v1.33.3) to remove sequences that contained ambiguous base calls and those that were longer/shorter than $90 \%$ of the sequences in a sample. As an additional precaution, sequences with high similarity to the PhiX genome were also removed using blastn (Altschul et al. 1990) (E-value $\left.<1 \times 10^{-5}\right)$. Preliminary analysis indicated minimal technical variation between sequencing runs, and duplicate samples were therefore merged prior to further analysis. After merging duplicates and filtering, samples contained at least 489,266 assembled sequences (average $1,209,197$ sequences).

Data were subsequently pooled across all samples, singletons (i.e. sequences appearing only once in the pooled dataset) were removed and the remaining data de-replicated to generate a set of unique sequences. Sequences representative of operational taxonomic units (OTUs) were then identified using the UPARSE pipeline (Edgar 2013) (usearch v8.0.1623), in which clustering was performed using the UPARSE-OTU algorithm and chimeras were identified using UCHIME in conjunction with the ChimeraSlayer reference database. All sequences were then assigned to an OTU using the USEARCH global algorithm (Edgar 2010) at a 97\% similarity threshold. On average, $83.5 \%$ of sequences in a sample were successfully assigned to an OTU. Sequences that could not be assigned to an OTU were assumed to represent sequencing/ assembly artefacts and were discarded from the study.

Taxonomic assignment of OTUs, OTU alignment and tree-building were performed using QIIME v1.9.0 (Caporaso et al. 2010b). The taxonomy of each OTU was determined using the RDP classifier v2.2 (Wang et al. 2007) in conjunction with the GreenGenes database, v13.5 (DeSantis et al. 2006) at a confidence threshold of 0.8. Representative OTU sequences were aligned using PyNAST (Caporaso et al. 2010a) and a neighbour-joining tree produced using FastTree (Price et al. 2009). Due to limitations in the ability of the V4 region to accurately identify closely related bacterial taxa, taxonomic classification of OTUs was restricted to the family level.

\section{Statistical analysis}

For all groups, $n=6$, with the exception of $\mathrm{C} 57 \mathrm{Bl} / 6$ mice fed Western diet, where $n=5$. Alpha diversity metrics Chao1 (Chao 1984) and phylogenetic distance (Faith \& Baker 2006) were calculated using QIIME and rarefaction curves indicated that the minimum sequencing depth (489,266 assembled sequences) was sufficient to accurately capture diversity (Supplementary Fig. 1, see section on supplementary data given at the end of this article). For all other analyses, following McMurdie and Holmes (2014), counts of sequences assigned to each OTU were normalised using the variance stabilising (vst) transformation in the $\mathrm{R}$ package DESeq2 (Love et al. 2014). Statistical comparison of microbial diversity between samples was calculated on Euclidean distance matrices of vst-transformed count 
data using the permutational multivariate analysis of variance (PERMANOVA) approach of Anderson (2001), implemented in the R package vegan (Oksanen et al. 2013).

To identify individual OTUs with clearly defined differences between diets, genotypes or a strong diet-genotype interaction, Euclidean distance-based PERMANOVA tests were repeated using each individual OTU as a univariate response variable. For OTUs showing significance for a particular model term $(P<0.01)$, effect sizes $\left(\eta^{2}\right)$ and partial effect sizes $\left(\eta_{\mathrm{p}}^{2}\right)$ were calculated from sums of squares (SS) to reflect the variance attributable to the relevant term as a proportion of variance within the model, where:

$$
\eta^{2}=\frac{S S_{\text {term }}}{S S_{\text {total }}}
$$

and:

$$
\eta_{\mathrm{p}}^{2}=\frac{\mathrm{SS}_{\text {term }}}{\mathrm{SS}_{\text {term }}+\mathrm{SS}_{\text {residual }}}
$$

\section{Results}

DNA was extracted from caecal, and colon contents were collected from Hsd11b1 $1^{\text {Del1/De1l }}$ and C57Bl/6 control mice fed either chow or Western diet. High-throughput sequencing of the V4 region of the bacterial 16S rRNA gene resulted in an average of $1.2 \times 10^{6}$ amplicons per sample. Clustering amplicons at a $97 \%$ sequence similarity threshold identified 1152 operational taxonomic units (OTUs), of which 231 were present in all samples (Supplementary Fig. 2A). OTUs were assigned a taxonomic identity using public databases, and the relative abundance of reads assigned to each OTU was subsequently used as a basis on which to compare microbiome composition across samples.

\section{Changes in the gut microbiome as a consequence of diet and 11/-HSD1 activity}

First, we considered the effect of diet and genotype on the composition, richness and diversity of the gut
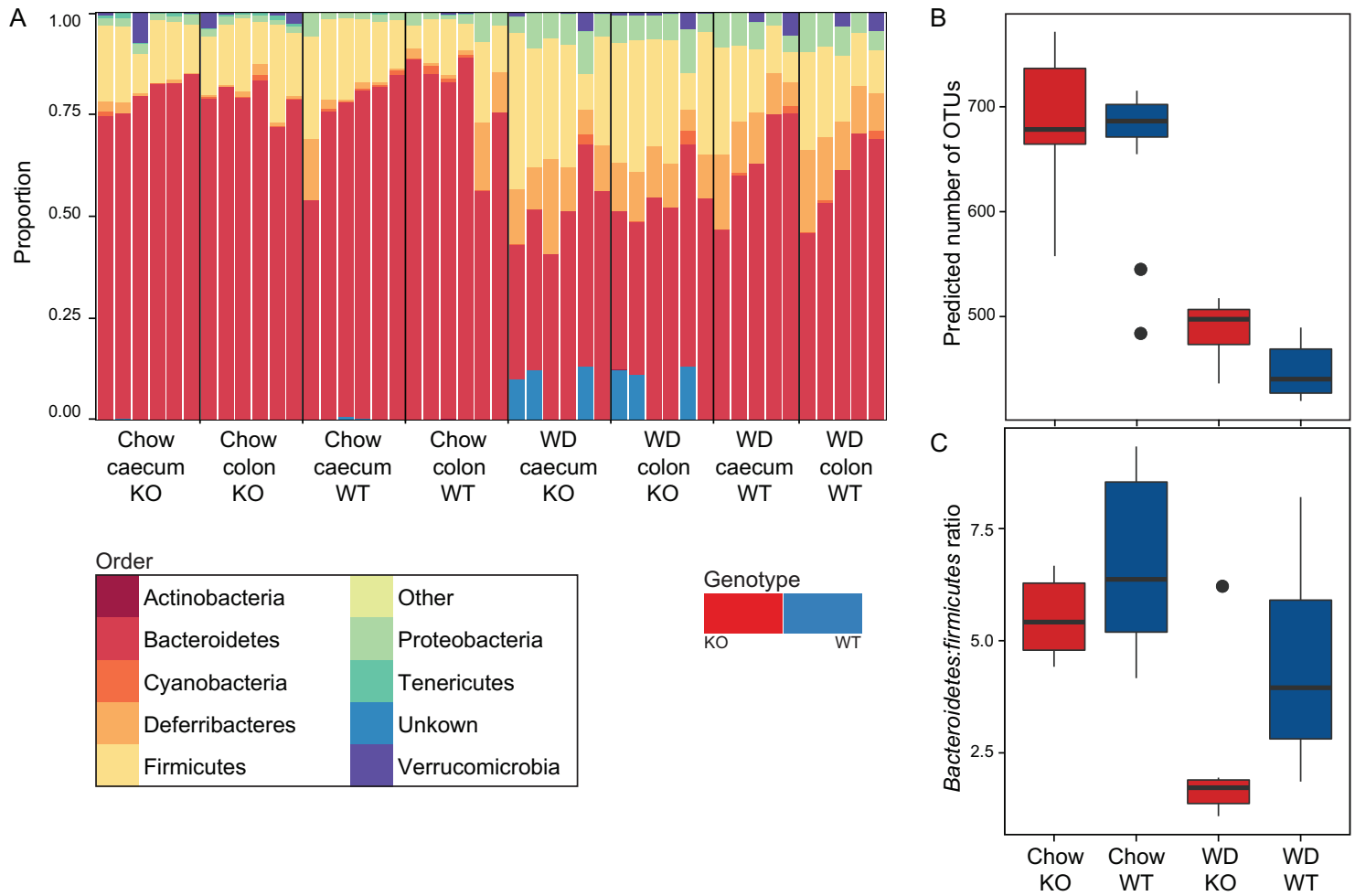

Figure 1

Gut microbiota of wild-type and Hsd11b1Del/1De1l mice fed Western and chow diets. (A) The relative proportion of bacterial sequence belonging to each of the dominant phyla detected in this study in Hsd11b1Del/1Del/ (KO) and C57Bl/6 control (WT) mice. As no difference was observed between caecum and colon, these samples are merged in subsequent figures. (B) Chao1 estimates of the number of unique operational taxonomic units (OTUs) in the gut microbiome of Hsd11 b1Del/1Del1 (KO, red bars) and C57BI/6 control (WT, blue bars) mice under different experimental conditions. Chao1 estimates were calculated after randomly down-sampling data so that all samples contain the same total number of OTU counts. (C) Ratio of the phyla Bacteroidetes and Firmicutes within each sample under different experimental conditions. Ratios were calculated on untransformed data.

http://joe.endocrinology-journals.org DOI: 10.1530/JOE-16-0578 (c) 2017 The authors Printed in Great Britain
Published by Bioscientifica Ltd 
Table 1 Results of permutational multivariate analysis of variance (PERMANOVA) test comparing composition of the gut microbiota across diets (chow vs Western) and genotypes (C57BI/6 vs Hsd11b1Del1/Del1). The test was based on vsttransformed counts of OTU abundance. For all groups, $n=6$, with the exception of C57BI/6 mice fed Western diet, where $n=5$.

\begin{tabular}{|c|c|c|c|c|c|}
\hline & $D f$ & Sums of Sqs & Mean Sqs & $\boldsymbol{F}$ & $\boldsymbol{P}$ \\
\hline Diet & 1 & 107,517 & 107,517 & 35.64 & 0.00001 \\
\hline Genotype & 1 & 22,727 & 22,727 & 7.534 & 0.0014 \\
\hline Diet $\times$ genotype & 1 & 17,496 & 17,496 & 5.80 & 0.0027 \\
\hline Residual & 19 & 57,318 & 3017 & 0.28 & \\
\hline
\end{tabular}

microbiome. In mice fed a chow diet, the microbiome was dominated by bacteria belonging to the phyla Bacteroidetes and Firmicutes (Fig. 1A). Richness estimates for the chow diet indicated the presence of around 500-800 OTUs (median 690 OTUs, Fig. 1B). Consistent with previous studies (Kim et al. 2012), a Western diet increased the relative abundance of bacteria belonging to the phyla Proteobacteria, Deferribateres and Firmicutes (Hildebrandt et al. 2009) and significantly decreased both richness (median 475 OTUs, ANOVA, $F=185.56, P<0.01$, Fig. 1B) and the Bacteroidetes:Firmicutes ratio (ANOVA, $F=11.90, P=0.03$, Fig. $1 \mathrm{C})$.

As observed previously, diet exerted a greater effect on composition of the gut microbiome than genotype (Carmody et al. 2015). Nonetheless, 11 $\beta$-HSD1 deficiency resulted in an increase in the predicted number of OTUs (ANOVA, $F=5.16, P=0.03$, Fig. 1B) and a decrease in the ratio of Bacteroidetes:Firmicutes (ANOVA, $F=4.39, P=0.05$,
Fig. 1C). Further changes in taxonomic abundance were observed at the family level (Supplementary Fig. 2). On a Western diet, 11 $\beta$-HSD1 deficiency was associated with a number of OTUs that could not be confidently assigned to any phylum. These OTUs were not present in all samples, highlighting the potential for inter-individual variability in microbiome composition.

Statistical comparison of OTU diversity across samples was carried out using permutational multivariate analysis of variance (PERMANOVA). No significant effect of sample origin (caecum vs colon) was observed $($ Pperm $=0.51)$. Accordingly, to avoid risk of type I error arising from repeated sampling from the same individual, data from caecal and colon samples for each animal were pooled to test for the effects of diet and genotype on diversity.

Statistical analysis showed that both diet and genotype significantly altered the diversity of the gut microbiome (Pperm $<0.01$, Table 1). Moreover, PERMANOVA tests revealed a significant interaction term, indicating that the effect of genotype was dependent upon the diet (Pperm $<0.01$, Table 1). Principal components analysis (PCA) revealed that diet exerted the strongest influence on microbial diversity, accounting for $59 \%$ of the observed variance in OTU abundance (PC1, Fig. 2A). Subsequent principal component axes indicated that genotype exerted a strong influence on microbial diversity, with $19 \%$ of the variation in diversity explained by differences between Hsd11b1Del1/De1l and control microbiomes in mice fed a chow or Western diet (PC2 and PC3, respectively, Fig. 2B).
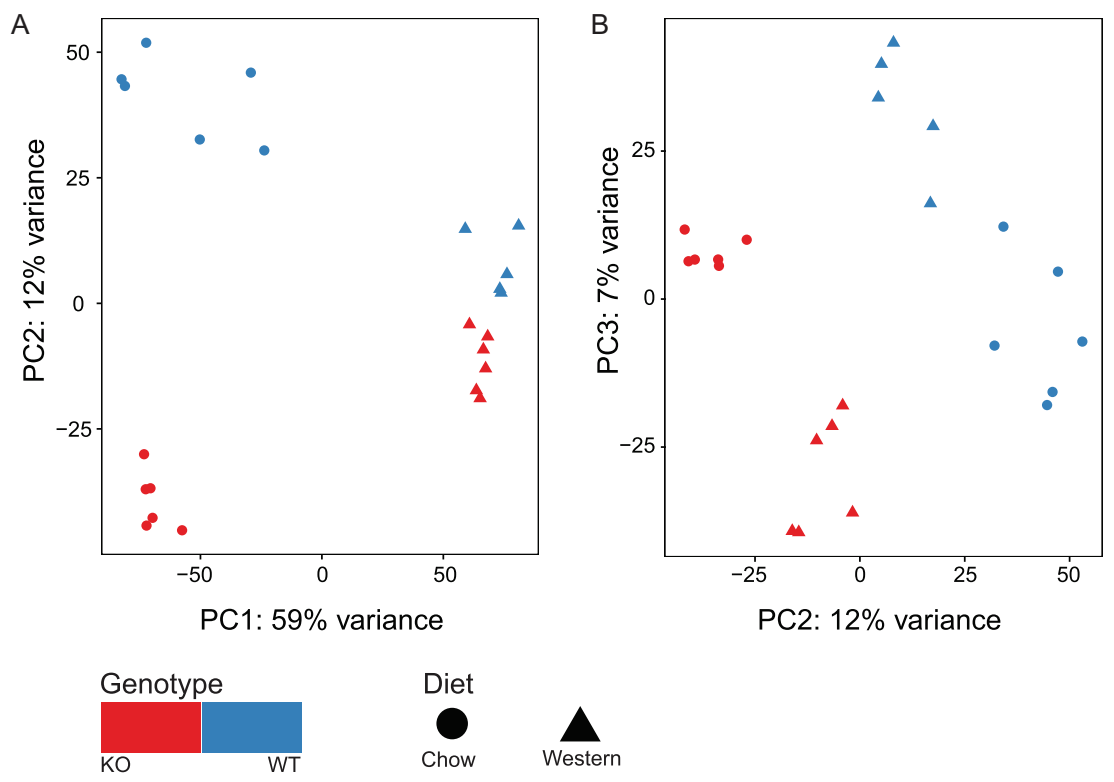

Figure 2

Principal components analysis (PCA) ordination showing the effect of diet and $11 \beta$-HSD 1 activity on the composition of the gut microbiota. Plots show (A) the first, second and (B) second and third axes of a PCA based on the abundance of the 1152 OTUs detected in this study. Red symbols: Hsd11b1De/1/De/1 (KO) mice, blue symbols: C57BI/6 control (WT) mice. For all groups, $n=6$, with the exception of C57BI/6 mice fed Western diet, where $n=5$.
() 2017 The authors Printed in Great Britain
Published by Bioscientifica Ltd. 


\section{Identifying bacterial taxa whose abundance is dependent on diet and 11 $\beta$-HSD1 activity}

Next, we sought to identify the main bacterial taxa responsible for driving differences in diversity that resulted from changing diet or 11 $\beta$-HSD1 activity. Effect sizes were calculated (see 'Methods' section) and for each OTU, they were used to represent the proportion of observed variation in abundance that could be explained by diet, genotype or a diet $\times$ genotype interaction (Supplementary Fig. 3). The $25 \%$ of OTUs with the largest effect size for a particular model term are depicted in Fig. 3.

Consistent with multivariate analysis, effect sizes $\left(\eta^{2}\right)$ demonstrated that diet frequently accounted for a greater proportion of variation in individual OTU abundance than either genotype or a diet $\times$ genotype interaction (Fig. 3A, B and C). Of those OTUs that significantly (Pperm<0.01) altered abundance in response to diet, the majority (494 out of 651) showed a decrease in relative abundance on the Western diet (Fig. 3A). This was evident for a number of OTUs belonging to the families S4-7, Prevotellaceae, Ruminococcaceae and Lachnospiraceae. However, the opposite was observed for OTUs belonging to the families Deferribacteraceae and Porphyromonadaceae, as well as a small number of OTUs belonging to the family $S 24-7$.

Genotype had a significant effect on 327 OTUs (Pperm $<0.01)$, and this effect was less one-sided (Fig. 3B), with some families, such as the Anaeroplasmataceae and Prevotellaceae, showing greater relative abundance in Hsd11b1Del1/De1l microbiomes, and others, such as the Rikenellaceae, showing greater relative abundance in wild-type microbiomes. Notably, many families affected by genotype showed changes in abundance that were moderated by diet, indicating that the effect of $11 \beta$-HSD1 activity on individual taxa was diet dependent. When directly considering OTUs for which an interaction between diet and genotype altered relative abundance (Fig. 3C), effect sizes indicated that this interaction had strongest explanatory power for bacteria belonging to the family Bacteroidaceae.

To investigate further how diet affected the microbiome response to 11ß-HSD1 deficiency, we used Pearson's method to correlate changes in the relative abundance of each OTU showing a significant diet $\times$ genotype interaction with the position of samples along the PC axes separating control and Hsd11b1 $1^{\text {Del1/Dell }}$ microbiomes on different diet backgrounds (as depicted in PC2 and PC3 in Fig. 2B). As expected, the majority of OTUs gave a strong Pearson's $r$ score, demonstrating strong correlation with one or the other of the explanatory PC axes (Fig. 4). Notably, however, overlaying information about taxonomic classification showed that many OTUs belonging to the families Prevotellaceae and Paraprevotellaceae displayed greater relative abundance in $H s d 11$ b1 $1_{\text {Del1/Dell microbiomes }}$ and $\mathrm{C} 57 \mathrm{Bl} / 6$ microbiomes, respectively, on a chow diet background. By contrast, OTUs belonging to the families Bacteroidaceae and Rikenellaceae displayed greater relative abundance in Hsd11b1Del1/De1l microbiomes and C57Bl/6 microbiomes, respectively, on a Western diet background. Although most OTUs showed changes in relative abundance in response to altered diet/genotype that were consistent with other members of their family, several
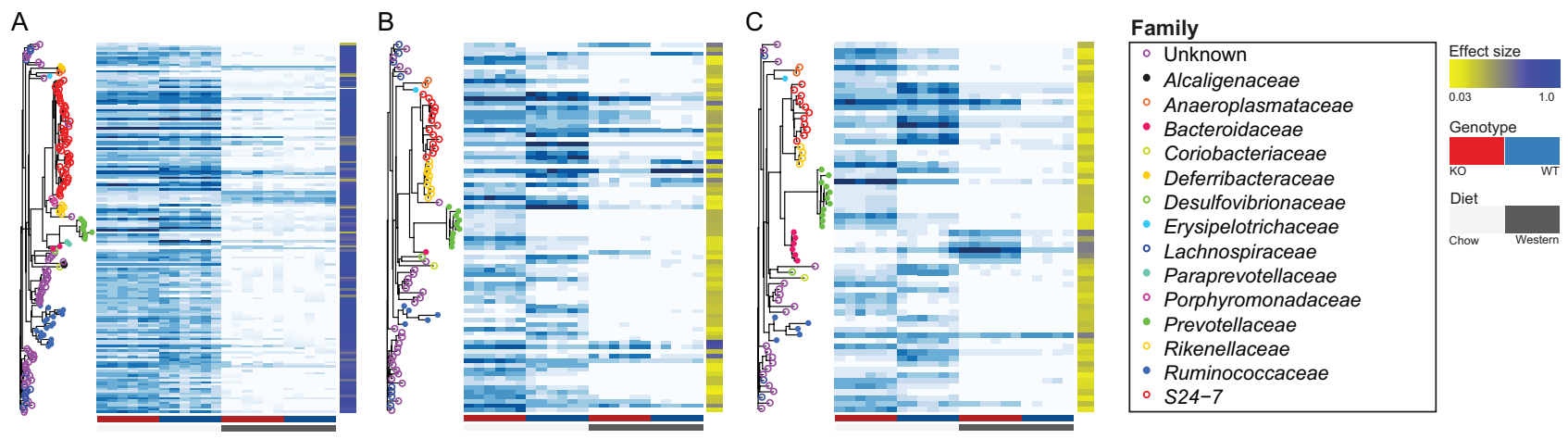

Figure 3

Heat maps depicting the relative abundance of OTUs for which (A) variation in abundance is dependent on diet, (B) variation in abundance is dependent on genotype, (C) variation in abundance as a consequence of genotype is dependent on diet (i.e. a diet $\times$ genotype interaction). For each contrast, data are only shown for the top $25 \%$ of significant OTUs ranked by the partial effect size $\left(\eta_{\mathrm{p}}{ }^{2}\right)$ of the relevant model term in univariate analysis of variance tests for differences in abundance between experimental conditions. Phylograms to the left of each heat map depict the phylogenetic relatedness between OTUs. Colour scales to the right of each OTU depict the proportion of the total variance $\left(\eta^{2}\right)$ that could be explained by the relevant model term. Colour bars beneath the heat maps indicate genotype (Hsd11b1Del/1Del1 (KO) mice: red, C57BI/6 control (WT) mice: blue) and diet (chow: light grey, Western: dark grey). For all groups, $n=6$, with the exception of C57Bl/6 mice fed Western diet, where $n=5$.

http://joe.endocrinology-journals.org

DOI: 10.1530/JOE-16-0578
(C) 2017 The authors Printed in Great Britain
Published by Bioscientifica Ltd 


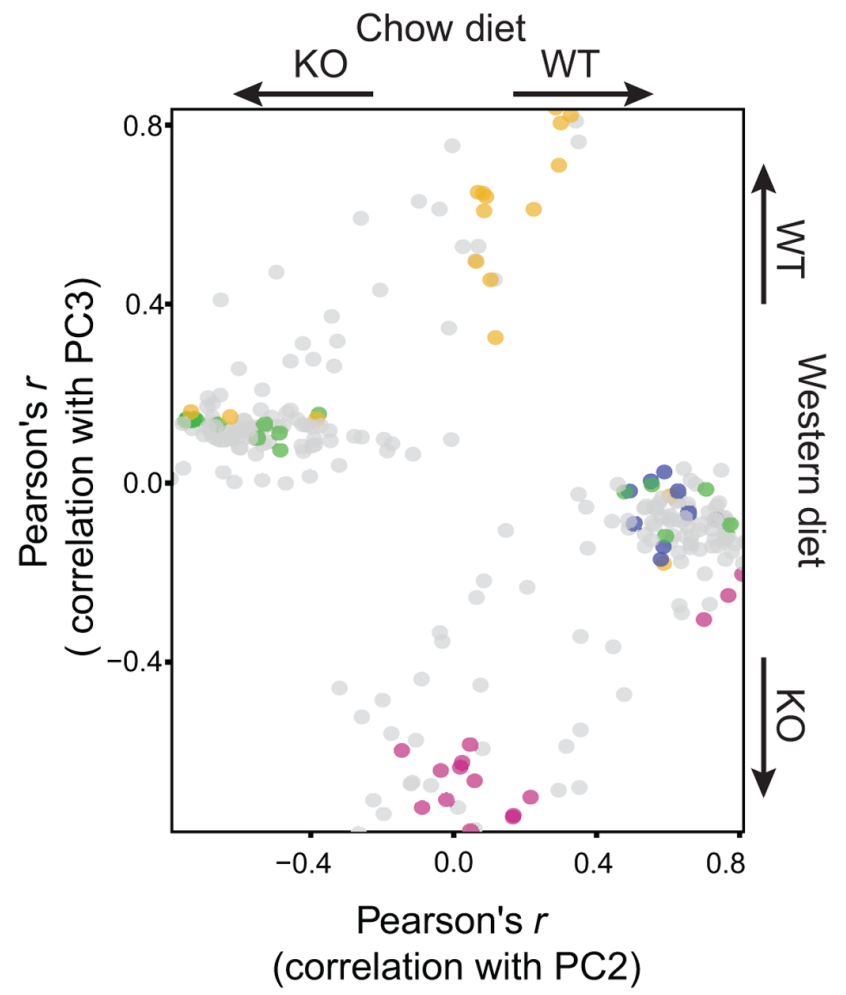

Rikenellaceae Paraprevotellaceae Bacteroidaceae Prevotellaceae Other Families

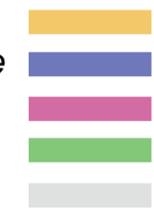

Figure 4

Bacterial taxa responsible for differences in diversity between the gut microbiota of C57BI/6 (WT) and Hsd11b1Del1/De1/ (KO) mice on different diet backgrounds. Each data point represents a single OTU and data are only shown for OTUs that have a significant diet $\times$ genotype interaction $(P<0.01)$ in univariate analysis of variance tests for differences in abundance between experimental conditions. The $x$ axis shows the Pearson correlation between the abundance of an OTU and the position of samples along the principal component axis in Fig. 2 that separates C57BI/6 and Hsd11b1Del1/De1/ mice on a chow diet (PC2). The $y$ axis shows the Pearson correlation between the abundance of an OTU and the position of samples along the principal component axis in Fig. 2 that separates C57BI/6 and Hsd11 b1Del1/De1/ mice on a Western diet (PC3). A strong positive correlation indicates that an OTU contributes to the diversity that distinguishes WT mice, a strong negative correlation indicates that an OTU contributes to the diversity that distinguishes $\mathrm{KO}$ mice. The colour of each data point depicts families that show strong trends that are restricted to different diet backgrounds. Changes in the relative abundance of these families in response to diet and $11 \beta$-HSD1 deficiency can be seen in Supplementary Fig. 2B. For all groups, $n=6$, with the exception of C57BI/6 mice fed Western diet, where $n=5$.

showed alternative patterns of abundance. For example, three OTUs belonging to the family Bacteroidaceae showed greater relative abundance in wild-type mice on a chow diet background, whereas the majority of OTUs belonging to this family showed altered response to genotype only on a Western diet background. Such patterns of abundance potentially reflect functional divergence of taxa below the family level, not detected in this study because of the limited taxonomic resolution provided by sequencing the V4 region. Taken together, however, these results demonstrate that $11 \beta$-HSD1 activity exerts an effect on the abundance of discrete bacterial taxa that is diet dependent and broadly consistent at family level.

\section{Discussion}

Here, we demonstrate that the enzyme 11 $\beta$-HSD1 acts to alter the composition of the mouse gut microbiome, and does so in a diet-dependent manner. Most notably, we show that $11 \beta$-HSD1 deficiency increases the relative abundance of the families Prevotellaceae on a chow diet and Bacteroidaceae on a Western diet. These families contain genera associated with inflammatory and cardiovascular disease, respectively. They also contain the principal genera that define common bacterial profiles (enterotypes) found within the human gut (Arumugam et al. 2011), which may themselves be diet related (Wu et al. 2011).

The family Prevotellaceae is associated with inflammatory disease in both humans and mouse models. In a study of patients with new-onset untreated rheumatoid arthritis, the presence of Prevotella copri correlated strongly with disease occurrence (Scher et al. 2013). We have previously reported that chow-fed $11 \beta$-HSD1-deficient mice show increased severity of acute inflammation, including in an experimental model of arthritis (Coutinho et al. 2012). Our findings here raise the possibility that this is related to the increased relative abundance of bacteria belonging to the Prevotellaceae in their gut microbiota. P. copri is also associated with more severe inflammation in animal models of colitis. Mice colonised with $P$. copri showed more severe colitis when exposed to dextran sulfate sodium (DSS) (Scher et al. 2013), probably mediated via induction of the cytokine CCL5, which exaggerates DSS-induced colitis (Elinav et al. 2011). 11 -HSD1 gene expression is increased in rodents during DSS-induced colitis and also in patients with inflammatory bowel disease (Zbankova et al. 2007, Stegk et al. 2009). However, this is interpreted as an antiinflammatory mechanism to increase local glucocorticoid exposure, and whether reduced expression or inhibition of 11 $\beta$-HSD1 can predispose to colitis has not been tested.

A high-fat/cholesterol diet profoundly alters the gut microbiota (Hildebrandt et al. 2009, David et al. 2014). High-fat/cholesterol diets also induce intestinal

Published by Bioscientifica Ltd 
inflammation, which is dependent upon the gut microbiota (Ding et al. 2010, Kim et al. 2012, Progatzky et al. 2014) and, at least for high-fat diet, has been associated with a decrease in the Bacteroidetes-to-Firmicutes ratio (Kim et al. 2012). Here, we show a dramatic alteration in the gut microbiota in mice fed a high-fat Western diet, including an increase in the Bacteroidetes-to-Firmicutes ratio that is consistent with the previous data. In addition, we show a smaller, but nonetheless significant, effect of genotype. Interestingly, and in contrast to chow-fed mice, Western diet-fed 11/-HSD1-deficient mice show a higher relative abundance of the family Bacteroidaceae. Whether this is beneficial or not remains to be determined. However, we have previously reported that $11 \beta$-HSD 1 deficient mice fed a high-fat diet show reduced inflammatory signalling in mesenteric adipose tissue, a depot that contains the lymph nodes that drain the colon (Wamil et al. 2011), suggesting a lower level of intestinal inflammation. In the future, it will be important to determine if the altered gut microbiota observed in Western diet-fed 11ß-HSD1deficient mice elicits milder intestinal inflammation.

Recent, large-scale studies of human cohorts have indicated that the presence and increased relative abundance of Bacteroidaceae (including the genus Bacteroides) is associated with a significant decrease in plasma triglyceride levels (Fu et al. 2015). Relative abundance of Bacteroides also correlates negatively with body mass index (Goodrich et al. 2014, Fu et al. 2015). Intriguingly, 11 $\beta$-HSD1 deficiency reduces plasma triglyceride levels and attenuates weight gain in mice fed a high-fat diet (Morton et al. 2004). We have also reported that $11 \beta-H S D 1$ deficiency reduces plasma triglyceride levels and is protective against atherosclerosis in Western diet-fed atherosclerosis-prone $\mathrm{Apoe}^{-/-}$mice (Kipari et al. 2013). However, in a similar study using a different line of $11 \beta$-HSD1 knockout mice, the reduction in plasma triglyceride levels was only observed in female mice (García et al. 2013). Nevertheless, the coincidence of 11 $\beta$-HSD1 deficiency, Bacteroidaceae abundance and reduced plasma triglyceride levels highlights a potential causative relationship that is deserving of further study.

The mechanisms that underlie the diet-specific effects of 11 $\beta$-HSD1 deficiency upon the gut microbiome are currently unclear. However, two possibilities are likely: alterations in glucocorticoid signalling or alterations in bile acid composition and/or signalling. 11ß-HSD1 is an important modulator of intracellular glucocorticoid levels, with glucocorticoids being one of the most potent regulators of immune cell function and phenotype. $11 \beta$-HSD1 is expressed and functional in a variety of immune cells including macrophages (Gilmour et al. 2006, Zhang \& Daynes 2007), dendritic cells (Freeman et al. 2005, Soulier et al. 2013), mast cells (Coutinho et al. 2013) and lymphocytes (Zhang et al. 2005), any of which could contribute to tolerance of gut microbes (Mortha et al. 2014). Altered intracellular glucocorticoid levels as a result of 11ß-HSD1 may therefore alter the local immune environment within the gut, with differential consequences for the microbiome, dependent upon diet. Tissue-specific disruption of $H s d 11 b 1$ in these cell types could address the possible role of $11 \beta$-HSD1 in tolerance to gut microbes.

11 $\beta$-HSD1 may also mediate host-microbiome interactions in a diet-specific manner via its role in bile acid homeostasis. High-fat diets both increase the quantity and alter the composition of bile acids in the gut (Ridlon et al. 2014), and bile acids have antimicrobial properties that make them important regulators of the gut microbiome (Begley et al. 2005). High-fat diets can therefore promote expansion of bile-tolerant bacteria, for example Bacteroides, which may in turn alter the synthesis of secondary bile acids. Microbial metabolism of bile acids in the intestines is a major determinant of bile acid pool size and composition (Begley et al. 2005, Sayin et al. 2013). Previously, we have reported preliminary data showing that 11/-HSD1 deficiency in mice impairs post-prandial bile acid release and alters the profile of bile acids in bile fluid, with a switch from the predominance of $7 \beta$-hydroxylated to $7 \alpha$-hydroxylated bile acids (Opiyo et al. 2014). Whether $11 \beta$-HSD1 itself directly converts $7 \alpha$-hydroxylated to $7 \beta$-hydroxylated bile acids is currently unknown, but several oxysterols with a keto moiety at the 7 position on the B ring are substrates for $11 \beta$-HSD1 (Odermatt \& Nashev 2010). Importantly, this includes the secondary bile acid 7-oxolithocholic acid (7-oxoLCA), converted by $11 \beta-H S D 1$ into chenodeoxycholic acid (CDCA) (Odermatt et al. 2011), a potent activator of the nuclear bile acid receptor, also known as the farnesoid $\mathrm{X}$ receptor (FXR). It is therefore plausible that $11 \beta$-HSD1 influences the composition of the gut microbiome by altering bile acid profile via its role in the synthesis of $7 \alpha$-hydroxylated bile acids. This merits future investigation.

In conclusion, we show that the effect of $11 \beta-H S D 1$ activity on the gut microbiome is diet dependent and alters the relative abundance of bacteria relevant to human clinical studies. Two plausible mechanisms for 11/-HSD1 mediation of host-microbiome interaction involve immune system regulation and bile acid homeostasis, and further functional studies are required to reveal the precise role of the gut microbiome in shaping the 11ß-HSD1

Published by Bioscientifica Ltd. 
deficiency phenotype. With the development of selective inhibitors of $11 \beta$-HSD1 for the treatment of metabolic disease and age-related cognitive decline (Anderson \& Walker 2013, Chapman et al. 2013), our studies could provide new insights into how diet may influence the outcome of therapeutic 11ß-HSD1 inhibition.

\section{Supplementary data}

This is linked to the online version of the paper at http://dx.doi.org/10.1530/ JOE-16-0578.

\section{Declaration of interest}

J R S holds patents on selective $11 \beta$-HSD1 inhibitors.

\section{Funding}

This work was supported by a Wellcome Trust Programme grant (083184; J R S, K E C). M N O was supported by a scholarship from the College of Medicine and Veterinary Medicine, University of Edinburgh. Edinburgh Genomics (University of Edinburgh) is partly supported through core grants from NERC (R8/H10/56), MRC (MR/K001744/1) and BBSRC (BB/J004243/1). The Computational Genomics Analysis and Training Centre (CGAT) was supported by the Medical Research Council (G1000902).

\section{Author contribution statement}

K E C and A H contributed to study conception; J S J, A H, K E C, K G and J R S designed the study. J S J, M N O and M T executed the study. J S J, $\mathrm{A} \mathrm{H}$ and $\mathrm{KEC}$ contributed to interpretation and $\mathrm{J} \mathrm{S} \mathrm{J}, \mathrm{KEC}$ and $\mathrm{A} \mathrm{H}$ prepared the manuscript.

\section{Acknowledgements}

The authors are grateful to staff at the University of Edinburgh Biomedical Research Resources facility for assistance with animal breeding and care. They would like to thank colleagues within the Centre for Cardiovascular science for helpful advice and discussion.

\section{References}

Altschul SF, Gish W, Miller W, Myers EW \& Lipman DJ 1990 Basic local alignment search tool. Journal of Molecular Biology 215 403-410. (doi:10.1016/S0022-2836(05)80360-2)

Anderson A \& Walker BR 2013 11ß-HSD1 inhibitors for the treatment of type 2 diabetes and cardiovascular disease. Drugs 73 1385-1393. (doi:10.1007/s40265-013-0112-5)

Anderson MJ 2001 A new method for non-parametric multivariate analysis of variance. Austral Ecology 26 32-46. (doi:10.1111/j.1442-9993.2001.tb00081.x)

Arumugam M, Raes J, Pelletier E, Le Paslier D, Yamada T, Mende DR, Fernandes GR, Tap J, Bruls T, Batto JM, et al. 2011 Enterotypes of the human gut microbiome. Nature $\mathbf{4 7 3}$ 174-180. (doi:10.1038/ nature09944)

Begley M, Gahan CG \& Hill C 2005 The interaction between bacteria and bile. FEMS Microbiology Reviews 29 625-651. (doi:10.1016/j. femsre.2004.09.003)

Brown JM \& Hazen SL 2015 The gut microbial endocrine organ: bacterially derived signals driving cardiometabolic diseases.

http://joe.endocrinology-journals.org

DOI: $10.1530 / J O E-16-0578$
(C) 2017 The authors Printed in Great Britain
Annual Review of Medicine 66 343-359. (doi:10.1146/annurevmed-060513-093205)

Buffie CG, Bucci V, Stein RR, McKenney PT, Ling L, Gobourne A, No D, Liu H, Kinnebrew M, Viale A, et al. 2015 Precision microbiome reconstitution restores bile acid mediated resistance to Clostridium difficile. Nature 517 205-208. (doi:10.1038/nature13828)

Buhler H, Perschel FH, Fitzner R \& Hierholzer K 1994 Endogenous inhibitors of $11 \beta$-OHSD - existence and possible significance. Steroids 59 131-135. (doi:10.1016/0039-128X(94)90090-6)

Caporaso JG, Bittinger K, Bushman FD, DeSantis TZ, Andersen GL \& Knight R 2010 a PyNAST: a flexible tool for aligning sequences to a template alignment. Bioinformatics 26 266-267. (doi:10.1093/ bioinformatics/btp636)

Caporaso JG, Kuczynski J, Stombaugh J, Bittinger K, Bushman FD, Costello EK, Fierer N, Pena AG, Goodrich JK, Gordon JI, et al. $2010 b$ QIIME allows analysis of high-throughput community sequencing data. Nature Methods 7 335-336. (doi:10.1038/nmeth.f.303)

Caporaso JG, Lauber CL, Walters WA, Berg-Lyons D, Huntley J, Fierer N, Owens SM, Betley J, Fraser L, Bauer M, et al. 2012 Ultra-highthroughput microbial community analysis on the Illumina HiSeq and MiSeq platforms. ISME Journal 6 1621-1624. (doi:10.1038/ ismej.2012.8)

Carmody RN, Gerber GK, Luevano JM Jr, Gatti DM, Somes L, Svenson KL \& Turnbaugh PJ 2015 Diet dominates host genotype in shaping the murine gut microbiota. Cell Host and Microbe 17 72-84. (doi:10.1016/j.chom.2014.11.010)

Chao A 1984 Non-parametric estimation of the number of classes in a population. Scandinavian Journal of Statistics $11265-270$.

Chapman K, Holmes M \& Seckl J 2013 11 $\beta$-Hydroxysteroid dehydrogenases: intracellular gate-keepers of tissue glucocorticoid action. Physiological Reviews 93 1139-1206. (doi:10.1152/ physrev.00020.2012)

Coutinho AE, Gray M, Brownstein DG, Salter DM, Sawatzky DA, Clay S, Gilmour JS, Seckl JR, Savill JS \& Chapman KE 2012 $11 \beta$-Hydroxysteroid dehydrogenase type 1 , but not type 2 , deficiency worsens acute inflammation and experimental arthritis in mice. Endocrinology 153 234-240. (doi:10.1210/en.2011-1398)

Coutinho AE, Brown JK, Yang F, Brownstein DG, Gray M, Seckl JR, Savill JS \& Chapman KE 2013 Mast cells express 11 $\beta$-hydroxysteroid dehydrogenase type 1 : a role in restraining mast cell degranulation. PLoS ONE 8 e54640. (doi:10.1371/journal.pone.0054640)

David LA, Maurice CF, Carmody RN, Gootenberg DB, Button JE, Wolfe BE, Ling AV, Devlin AS, Varma Y, Fischbach MA, et al. 2014 Diet rapidly and reproducibly alters the human gut microbiome. Nature 505 559-563. (doi:10.1038/nature12820)

DeSantis TZ, Hugenholtz P, Larsen N, Rojas M, Brodie EL, Keller K, Huber T, Dalevi D, Hu P \& Andersen GL 2006 Greengenes, a chimera-checked $16 \mathrm{~S}$ rRNA gene database and workbench compatible with ARB. Applied and Environmental Microbiology 72 5069-5072. (doi:10.1128/AEM.03006-05)

Ding S, Chi MM, Scull BP, Rigby R, Schwerbrock NM, Magness S, Jobin C \& Lund PK 2010 High-fat diet: bacteria interactions promote intestinal inflammation which precedes and correlates with obesity and insulin resistance in mouse. PLoS ONE 5 e12191. (doi:10.1371/ journal.pone.0012191)

Edgar RC 2010 Search and clustering orders of magnitude faster than BLAST. Bioinformatics 26 2460-2461. (doi:10.1093/bioinformatics/ btq461)

Edgar RC 2013 UPARSE: highly accurate OTU sequences from microbial amplicon reads. Nature Methods 10 996-998. (doi:10.1038/ nmeth.2604)

Elinav E, Strowig T, Kau AL, Henao-Mejia J, Thaiss CA, Booth CJ, Peaper DR, Bertin J, Eisenbarth SC, Gordon JI, et al. 2011 NLRP6 inflammasome regulates colonic microbial ecology and risk for colitis. Cell 145 745-757. (doi:10.1016/j.cell.2011.04.022) 
Evans JM, Morris LS \& Marchesi JR 2013 The gut microbiome: the role of a virtual organ in the endocrinology of the host. Journal of Endocrinology 218 R37-R47. (doi:10.1530/JOE-13-0131)

Faith DP \& Baker AM 2006 Phylogenetic diversity (PD) and biodiversity conservation: some bioinformatics challenges. Evolutionary Bioinformatics Online 2 121-128.

Freeman L, Hewison M, Hughes SV, Evans KN, Hardie D, Means TK \& Chakraverty R 2005 Expression of 11 $\beta$-hydroxysteroid dehydrogenase type 1 permits regulation of glucocorticoid bioavailability by human dendritic cells. Blood 106 2042-2049. (doi:10.1182/blood-2005-010186)

Fu J, Bonder MJ, Cenit MC, Tigchelaar EF, Maatman A, Dekens JA, Brandsma E, Marczynska J, Imhann F, Weersma RK, et al. 2015 The gut microbiome contributes to a substantial proportion of the variation in blood lipids. Circulation Research 117 817-824. (doi:10.1161/CIRCRESAHA.115.306807)

García RA, Search DJ, Lupisella JA, Ostrowski J, Guan B, Chen J, Yang W-P, Truong A, He A, Zhang R, et al. 2013 11 $\beta$-Hydroxysteroid dehydrogenase type 1 gene knockout attenuates atherosclerosis and in vivo foam cell formation in hyperlipidemic Apoe-/- mice. PLoS ONE 8 e53192. (doi:10.1371/journal.pone.0053192)

Gilmour JS, Coutinho AE, Cailhier JF, Man TY, Clay M, Thomas G, Harris HJ, Mullins JJ, Seckl JR, Savill JS, et al. 2006 Local amplification of glucocorticoids by $11 \beta$-hydroxysteroid dehydrogenase type 1 promotes macrophage phagocytosis of apoptotic leukocytes. Journal of Immunology 176 7605-7611. (doi:10.4049/jimmunol.176.12.7605)

Giongo A, Gano KA, Crabb DB, Mukherjee N, Novelo LL, Casella G, Drew JC, Ilonen J, Knip M, Hyoty H, et al. 2011 Toward defining the autoimmune microbiome for type 1 diabetes. ISME Journal 5 82-91. (doi:10.1038/ismej.2010.92)

Goodrich JK, Waters JL, Poole AC, Sutter JL, Koren O, Blekhman R, Beaumont M, Van Treuren W, Knight R, Bell JT, et al. 2014 Human genetics shape the gut microbiome. Cell 159 789-799. (doi:10.1016/j. cell.2014.09.053)

Hildebrandt MA, Hoffmann C, Sherrill-Mix SA, Keilbaugh SA, Hamady M, Chen YY, Knight R, Ahima RS, Bushman F \& Wu GD 2009 High-fat diet determines the composition of the murine gut microbiome independently of obesity. Gastroenterology 137 1716-1724. (doi:10.1053/j.gastro.2009.08.042)

Islam KBMS, Fukiya S, Hagio M, Fujii N, Ishizuka S, Ooka T, Ogura Y, Hayashi T \& Yokota A 2011 Bile acid is a host factor that regulates the composition of the cecal microbiota in rats. Gastroenterology 141 1773-1781. (doi:10.1053/j.gastro.2011.07.046)

Kim KA, Gu W, Lee IA, Joh EH \& Kim DH 2012 High fat diet-induced gut microbiota exacerbates inflammation and obesity in mice via the TLR4 signaling pathway. PLOS ONE 7 e47713. (doi:10.1371/journal. pone.0047713)

Kipari T, Hadoke PW, Iqbal J, Man TY, Miller E, Coutinho AE, Zhang Z, Sullivan KM, Mitic T, Livingstone DE, et al. 2013 11ß-hydroxysteroid dehydrogenase type 1 deficiency in bone marrow-derived cells reduces atherosclerosis. FASEB Journal 27 1519-1531. (doi:10.1096/ fj.12-219105)

Love MI, Huber W \& Anders S 2014 Moderated estimation of fold change and dispersion for RNA-seq data with DESeq2. Genome Biology 15550. (doi:10.1186/s13059-014-0550-8)

McMurdie PJ \& Holmes S 2014 Waste not, want not: why rarefying microbiome data is inadmissible. PLoS Computational Biology $\mathbf{1 0}$ e1003531. (doi:10.1371/journal.pcbi.1003531)

McSweeney SJ, Hadoke PWF, Kozak AM, Small GR, Khaled H, Walker BR \& Gray GA 2010 Improved heart function follows enhanced inflammatory cell recruitment and angiogenesis in 11 $\beta$-HSD1deficient mice post-MI. Cardiovascular Research 88 159-167. (doi:10.1093/cvr/cvq149)

Mortha A, Chudnovskiy A, Hashimoto D, Bogunovic M, Spencer SP, Belkaid Y \& Merad M 2014 Microbiota-dependent crosstalk between macrophages and ILC3 promotes intestinal homeostasis. Science $\mathbf{3 4 3}$ 1249288. (doi:10.1126/science.1249288)

Morton NM, Paterson JM, Masuzaki H, Holmes MC, Staels B, Fievet C, Walker BR, Flier JS, Mullins JJ \& Seckl JR 2004 Novel adipose tissue-mediated resistance to diet-induced visceral obesity in 11/-hydroxysteroid dehydrogenase type 1-deficient mice. Diabetes 53 931-938. (doi:10.2337/diabetes.53.4.931)

Odermatt A \& Nashev LG 2010 The glucocorticoid-activating enzyme 11ß-hydroxysteroid dehydrogenase type 1 has broad substrate specificity: physiological and toxicological considerations. Journal of Steroid Biochemistry and Molecular Biology 119 1-13. (doi:10.1016/j. jsbmb.2010.01.007)

Odermatt A, Da Cunha T, Penno CA, Chandsawangbhuwana C, Reichert C, Wolf A, Dong M \& Baker ME 2011 Hepatic reduction of the secondary bile acid 7-oxolithocholic acid is mediated by 11ß-hydroxysteroid dehydrogenase 1. Biochemical Journal 436 621-629. (doi:10.1042/BJ20110022)

Oksanen J, Blanchet FG, Kindt R, Legendre P, Minchin PR, O'Hara RB, Simpson GL, Solymos P, Henry M, Stevens H, et al. 2013 vegan: Community Ecology Package. (available at: http://CRAN.R-project. org/package=vegan).

Opiyo NM, Escher G, Kenyon CJ, Seckl JR, Andrew R \& Chapman KE 2014 The role of glucocorticoid metabolism in bile acid homeostasis. Endocrine Abstracts 34 P252. (doi:10.1530/endoabs.34.p252)

Penno CA, Morgan SA, Vuorinen A, Schuster D, Lavery GG \& Odermatt A 2013 Impaired oxidoreduction by $11 \beta$-hydroxysteroid dehydrogenase 1 results in the accumulation of 7-oxolithocholic acid. Journal of Lipid Research 54 2874-2883. (doi:10.1194/jlr.M042499)

Penno CA, Morgan SA, Rose AJ, Herzig S, Lavery GG \& Odermatt A 2014 11 $\beta$-Hydroxysteroid dehydrogenase-1 is involved in bile acid homeostasis by modulating fatty acid transport protein- 5 in the liver of mice. Molecular Metabolism 3 554-564. (doi:10.1016/j. molmet.2014.04.008)

Perschel FH, Buhler H \& Hierholzer K 1991 Bile-acids and their amidates inhibit 11 $\beta$-hydroxysteroid dehydrogenase obtained from rat kidney. Pflugers Archiv: European Journal of Physiology 418 538-543. (doi:10.1007/BF00370568)

Price MN, Dehal PS \& Arkin AP 2009 FastTree: computing large minimum evolution trees with profiles instead of a distance matrix. Molecular Biology and Evolution 26 1641-1650. (doi:10.1093/molbev/msp077)

Progatzky F, Sangha NJ, Yoshida N, McBrien M, Cheung J, Shia A, Scott J, Marchesi JR, Lamb JR, Bugeon L, et al. 2014 Dietary cholesterol directly induces acute inflammasome-dependent intestinal inflammation. Nature Communications 5 5864. (doi:10.1038/ ncomms6864)

Ridlon JM, Kang DJ, Hylemon PB \& Bajaj JS 2014 Bile acids and the gut microbiome. Current Opinion in Gastroenterology 30 332-338. (doi:10.1097/MOG.0000000000000057)

Sayin SI, Wahlstrom A, Felin J, Jantti S, Marschall HU, Bamberg K, Angelin BHyotylainen T, Oresic M \& Backhed F 2013 Gut microbiota regulates bile acid metabolism by reducing the levels of tauro-betamuricholic acid, a naturally occurring FXR antagonist. Cell Metabolism 17 225-235. (doi:10.1016/j.cmet.2013.01.003)

Scher JU, Sczesnak A, Longman RS, Segata N, Ubeda C, Bielski C, Rostron T, Cerundolo V, Pamer EG, Abramson SB, et al. 2013 Expansion of intestinal Prevotella copri correlates with enhanced susceptibility to arthritis. eLife 2 e01202. (doi:10.7554/ elife.01202)

Schloss PD, Westcott SL, Ryabin T, Hall JR, Hartmann M, Hollister EB, Lesniewski RA, Oakley BB, Parks DH, Robinson CJ, et al. 2009 Introducing mothur: open-source, platform-independent, community-supported software for describing and comparing microbial communities. Applied and Environmental Microbiology $\mathbf{7 5}$ 7537-7541. (doi:10.1128/AEM.01541-09) http://joe.endocrinology-journals.org

DOI: $10.1530 / J O E-16-0578$
(C) 2017 The authors Printed in Great Britain
Published by Bioscientifica Ltd. 
Soulier A, Blois SM, Sivakumaran S, Fallah-Arani F, Henderson S, Flutter B, Rabbitt EH, Stewart PM, Lavery GG, Bennett C, et al. 2013 Cell-intrinsic regulation of murine dendritic cell function and survival by prereceptor amplification of glucocorticoid. Blood $\mathbf{1 2 2}$ 3288-3297. (doi:10.1182/blood-2013-03-489138)

Stegk JP, Ebert B, Martin HJ \& Maser E 2009 Expression profiles of human 11 $\beta$-hydroxysteroid dehydrogenases type 1 and type 2 in inflammatory bowel diseases. Molecular and Cellular Endocrinology 301 104-108. (doi:10.1016/j.mce.2008.10.030)

Tang SH, Silva FJ, Tsark WM \& Mann JR 2002 A Cre/loxP-deleter transgenic line in mouse strain 129S1/SvImJ. Genesis 32 199-202. (doi:10.1002/gene.10030)

Wamil M, Battle JH, Turban S, Kipari T, Seguret D, de Sousa Peixoto R, Nelson YB, Nowakowska D, Ferenbach D, Ramage L, et al. 2011 Novel fat depot-specific mechanisms underlie resistance to visceral obesity and inflammation in 11 $\beta$-hydroxysteroid dehydrogenase type 1-deficient mice. Diabetes 60 1158-1167. (doi:10.2337/db10-0830)

Wang Q, Garrity GM, Tiedje JM \& Cole JR 2007 Naive Bayesian classifier for rapid assignment of rRNA sequences into the new bacterial taxonomy. Applied and Environmental Microbiology 73 5261-5267. (doi:10.1128/AEM.00062-07)

White CI, Jansen MA, McGregor K, Mylonas KJ, Richardson RV, Thomson A, Moran CM, Seckl JR, Walker BR, Chapman KE, et al. 2015 Cardiomyocyte and vascular smooth muscle independent $11 \beta$-hydroxysteroid dehydrogenase 1 amplifies infarct expansion, hypertrophy and the development of heart failure following myocardial infarction in male mice. Endocrinology 157 346-357. (doi:10.1210/en.2015-1630)

Wu HJ \& Wu E 2012 The role of gut microbiota in immune homeostasis and autoimmunity. Gut Microbes 3 4-14. (doi:10.4161/gmic.19320)

Wu GD, Chen J, Hoffmann C, Bittinger K, Chen YY, Keilbaugh SA, Bewtra M, Knights D, Walters WA, Knight R, et al. 2011 Linking longterm dietary patterns with gut microbial enterotypes. Science $\mathbf{3 3 4}$ 105-108. (doi:10.1126/science.1208344)

Yokota A, Fukiya S, Islam KBMS, Ooka T, Ogura Y, Hayashi T, Hagio M \& Ishizuka S 2012 Is bile acid a determinant of the gut microbiota on a high-fat diet? Gut Microbes 3 455-459. (doi:10.4161/gmic.21216)

Zbankova S, Bryndova J, Leden P, Kment M, Svec A \& Pacha J 2007 11 $\beta$-Hydroxysteroid dehydrogenase 1 and 2 expression in colon from patients with ulcerative colitis. Journal of Gastroenterology and Hepatology 22 1019-1023. (doi:10.1111/j.14401746.2006.04529.x)

Zhang TY \& Daynes RA 2007 Macrophages from 11ß-hydroxysteroid dehydrogenase type 1-deficient mice exhibit an increased sensitivity to lipopolysaccharide stimulation due to TGF- $\beta$-mediated up-regulation of SHIP1 expression. Journal of Immunology 179 6325-6335. (doi:10.4049/jimmunol.179.9.6325)

Zhang TY, Ding X \& Daynes RA 2005 The expression of $11 \beta$-hydroxysteroid dehydrogenase type I by lymphocytes provides a novel means for intracrine regulation of glucocorticoid activities. Journal of Immunology 174 879-889. (doi:10.4049/ jimmunol.174.2.879)

Received in final form 16 November 2016 Accepted 24 November 2016 Accepted Preprint published online 24 November 2016 (c) 2017 The authors Printed in Great Britain
Published by Bioscientifica Ltd. 